\title{
EXPERIENCIAS
}

\section{DISEÑO IMPLEMENTACIÓN Y EVALUACIÓN DE UNA EXPERIENCIA MULTICULTURAL}

\author{
DESIGN, IMPLEMENTATION AND EVALUATION \\ OF A MULTICULTURAL EXPERIENCE
}

\author{
Anna Calsina*, Marta Liria*, Sandra Martínez Martínez* \\ Universidad de Barcelona
}

\begin{abstract}
RESUMEN
La experiencia se basó en la realización de una gimkana en un centro de educación no formal a un grupo de menores. (Intermón, 1991; Rodríguez, Ginés y Saliba,1995; Babí, García, Gipert y Rebojo, 1997). Para poder llevar a cabo dicha experiencia se ha seguido la estructura propuesta por Tort y Millán (2000).

A partir de los objetivos generales, entre otros, destacamos: Fomentar la cohesión y cooperación del grupo, y conocer algunas tradiciones y costumbres de las diferentes culturas. Utilizamos una serie de instrumentos para valorarlos que a pesar de no estar estandarizados fueron de gran utilidad pese a la dificultad que ha planteado la muestra. Algunas conclusiones son las siguientes: Se han logrado los objetivos aunque algunos sujetos mostraban cierta reticencia a integrarse en el grupo, por otra parte, las actividades de mayor esfuerzo intelectual eran las que presentaban una mayor dificultad a la hora de llevarlas a cabo.
\end{abstract}

Palabras clave: Multiculturalismo, juego, cuento, danza, tradición, diversidad, cooperación.

\begin{abstract}
The experience was based on holding a gymkhana, for a group of children, in an informal educational centre. (Intermón, 1991; Rodríguez, Ginés y Saliba, 1995; Babí, García, Gispert y Rebojo, 1997). In order to carry out the above mentioned experience the structure proposed by Tort and Millán (2000) was followed.

Taking the general objectives as a starting point, we can highlight: Encouraging the group to come together and to cooperate and getting to know different cultures' traditions and customs. We used a series of instruments to evaluate the objectives, which despite not being standardised were very useful

\footnotetext{
* Las autoras son colaboradoras de la asignatura de Pedagogía de la Inadaptación Social, desarrollan la línea de investigación sobre la Problemática de la Multiculturalidad en la Sociedad. Departamento de Métodos y Diagnóstico en Educación de la Facultad de Pedagogía. Universidad de Barcelona.
} 
even though the sample (the children) presented some difficulties. Some of the conclusions are the following: The objectives were achieved although some of the subjects showed a certain unwillingness to integrate into the group, on the other hand, the activities which required more intellectual effort were the ones which were the most difficult to carry out.

Key words: Multiculturalism, game, story, dance, tradition, diversity, cooperation.

\section{Planteamiento teórico}

Con la finalidad de llevar a cabo el proyecto, nos hemos basado en la estructura que proponen Tort y Millán (2000), así como otros autores como Palom y Tort (1991), García y Ramírez (1996) y Mille (1997).

Nuestro proyecto fue concebido con una idea lúdica, es decir a través del juego. Toman esta línea de trabajo Balboas (1995), Clapés et al. (1995) Marín et al. (1999) y Movibaix (1992).

Nuestra experiencia se basa en un viaje alrededor del mundo para conocer como es su gente, que diferencias existen entre unos y otros y cuales son sus tradiciones, entre ellas el cuento, trabajado por Intermón (1991), Rodríguez, Ginés y Saliba (1995), Babí, García, Gipert y Rebojo (1997), Lasa (1997), Intermón (1998), Luque y Fidel (1998), la Fundación Akwaba (1999), López (2000), Aula de Esplai del Vallès (2000) entre otros; la representación de una pequeña historia con sombras chinas, para ello podemos encontrar una maleta pedagógica realizada por Intermón (1991); la música y la danza, trabajado por Intermón (1988) y Giradles y Pelegrín (1996) y el aspecto culinario, en esta línea ha trabajado Coepfert (1995).

Babí et al (1997) desarrollaron otras actividades que también se pueden relacionar con nuestro proyecto "Volta per la diversitat del món», (Vuelta por la diversidad del mundo).

La sensibilización por la mejora de las relaciones interculturales y por el papel que deberían desarrollar las instituciones públicas con respecto al tema también se encuentra presente en otros autores como Roquer C, Roquer J, Tovías, Valcárcel, y Dahou (1997).

En cuanto a las líneas de trabajo nos basamos en el aprendizaje cooperativo, la participación activa y la corresponsabilidad como lo propone Aguilera et al. (1997).

Por otro lado, podemos comentar que SOS Racismo tiene un catálogo de actividades y materiales dentro del área pedagógica para trabajar la interculturalidad, contra el racismo y la xenofobia.

El proyecto que hemos diseñado y llevado a cabo lo hemos denominado con el título «Volta per la diversitat del món» (Vuelta por la diversidad del mundo). Este proyecto se plantea conseguir diferentes objetivos y lo hemos realizado con la finalidad de observar el colectivo magrebí del barrio de la Florida (l’Hospitalet, Barcelona) y más concretamente dentro del Casal «La Llumeneta». Este barrio está determinado por un continuo flujo migratorio y todas las consecuencias que esto conlleva. En estos últimos años ha incrementado el interés por el tema del multculturalismo, motivo por el cual el Casal comenzó a potenciar aspectos relacionados con el mismo.

El grupo diana está formado por 7 niños y niñas (3 niños y 4 niñas) entre los 8 y los 10 años (grupo de medianos-grandes), de los cuales la gran mayoría son hijos de inmigrantes marroquines o nacidos en Marruecos, la ratio educador-menor se sitúa en 1 educador por cada 4 menores. 
Como objetivos generales nos propusimos:

1. Fomentar la cohesión y cooperación del grupo mediante el diálogo y el respeto.

2. Reflexionar sobre la actitud que a menudo se muestra hacia otras culturas así como todo aquello que se ve como diferente a lo propio.

3. Conocer algunas tradiciones y costumbres de las diferentes culturas así como su localización originaria.

Los objetivos específicos vienen reflejados en la tabla 1 .

\section{Valoración}

Nuestra valoración se ha visto limitada por el escaso número de sujetos que contenía la muestra que participaba en la actividad, motivo por el cual nuestra apreciación no puede generalizarse.

El análisis de nuestra actividad, la hemos centrado en la información obtenida a través del Feed-back (Aguilera et al., 1997) y el diálogo continuado entre monitores y usuarios del casal, tratando de fomentar la colaboración en grupo y una actitud positiva en relación a la diversidad como la trabajan Babí, García, Gipert y Rebojo (1997) a través del cuento. A partir de esta información podemos deducir que:

- Los usuarios han dado una respuesta positiva hacia la actividad puesto que han colaborado de manera activa en la experiencia, actitud que también potencia Aguilera et al. (1997). Aún así, ha habido niños que han mostrado cierta reticencia, coincidiendo estos con los que menos tiempo llevan en el país, sobretodo en aquellas actividades que requerían una mayor atención cognitiva.

- Hemos intentado potenciar el trabajo cooperativo (gimkana) entre diversos grupos, puesto que consideramos que se trata de una base esencial para fomentar actitudes de respeto y tolerancia, hecho que también trabajaron Balboas (1995), Marín et al. (1999) y Fundación Akwaba (1999). Creemos que la formación en grupos nos ha ayudado a alcanzar con más facilidad los objetivos específicos propuestos, la valoración de éstos se recoge en la tabla 1.

Para poder llevar a cabo una buena valoración de los objetivos específicos nos hemos basado en los siguientes criterios:

- Para el objetivo específico 1.1:

- En el resultado final del cuento se ve reflejada alguna de las ideas que ha aportado el niño.

- El niño ha tenido en cuenta la opinión del resto de sus compañeros.

- Para el objetivo específico 1.2:

- El niño se ha implicado en la dramatización del cuento.

- El niño ha respetado el turno de palabra.

- Para el objetivo específico 2.1:

- El niño se ha mostrado abierto por aprender las distintas tradiciones.

- El niño se divierte en la realización de cada una de las actividades. 


\section{TABLA 1: Valoración cualitativa de los objetivos específicos propuestos.}

\begin{tabular}{|c|c|c|}
\hline Objetivos generales & Objetivos específicos & Valoración \\
\hline $\begin{array}{l}\text { 1. Fomentar la cohe- } \\
\text { sión y coopera- } \\
\text { ción del grupo } \\
\text { mediante el diálo- } \\
\text { go y el respeto. }\end{array}$ & $\begin{array}{l}\text { 1.1. Construir un cuento teniendo } \\
\text { en cuenta las ideas de los pro- } \\
\text { pios compañeros. } \\
\text { 1.2. Dramatizar un cuento adoptan- } \\
\text { do los roles de los diferentes } \\
\text { personajes con la finalidad de } \\
\text { trasladarlo a la vida cotidiana. }\end{array}$ & $\begin{array}{l}\text { - El cuento final ha sido resultado } \\
\text { del conjunto de ideas aportadas } \\
\text { por todos los miembros del gru- } \\
\text { po. Aunque en uno de los grupos } \\
\text { se detectó que uno de los niños } \\
\text { actuaba como líder, impidiendo } \\
\text { así la participación de todos los } \\
\text { miembros por igual. }\end{array}$ \\
\hline $\begin{array}{l}\text { 2. Reflexionar sobre } \\
\text { la actitud que a } \\
\text { menudo se mues- } \\
\text { tra hacia otras } \\
\text { culturas así como } \\
\text { todo aquello que } \\
\text { se ve como dife- } \\
\text { rente a lo propio. }\end{array}$ & $\begin{array}{l}\text { 2.1. Valorar positivamente las tra- } \\
\text { diciones que se muestran a lo } \\
\text { largo de la gimkana. } \\
\text { 2.2. Recibir y despedir hospitala- } \\
\text { riamente al personaje invitado. }\end{array}$ & $\begin{array}{l}\text { - Se ha detectado una aceptación } \\
\text { positiva hacia el personaje (extra- } \\
\text { terrestre), invitándolo a quedarse } \\
\text { a vivir en nuestro planeta y ofre- } \\
\text { ciéndole un lugar donde vivir. } \\
\text { - En el conjunto de pruebas realiza- } \\
\text { das a lo largo de la gimkana, los } \\
\text { niños no han mostrado rechazo } \\
\text { por aprender y conocer las dife- } \\
\text { rentes tradiciones que en ella se } \\
\text { han expuesto. }\end{array}$ \\
\hline $\begin{array}{l}\text { 3. Conocer algunas } \\
\text { tradiciones y cos- } \\
\text { tumbres de las di- } \\
\text { ferentes culturas } \\
\text { así como locali- } \\
\text { zación originaria. }\end{array}$ & $\begin{array}{l}\text { 3.1. Situar y pintar los diferentes } \\
\text { continentes en un mapa pro- } \\
\text { porcionado a cada grupo. } \\
\text { 3.2. Representar las diferentes dan- } \\
\text { zas del continente americano. } \\
\text { 3.3. Elaborar una receta culinaria } \\
\text { europea. } \\
\text { 3.4. Inventar, mediante sombras } \\
\text { chinas, una fábula. }\end{array}$ & $\begin{array}{l}\text { - En el conjunto de los mapas se ha } \\
\text { podido observar que los sujetos } \\
\text { de la muestra han situado correc- } \\
\text { tamente los diferentes continentes } \\
\text { pintándolos del color identificato- } \\
\text { rio de cada uno. } \\
\text { - Al final de la prueba, los niños han } \\
\text { enseñado al resto de los compañe- } \\
\text { ros el conjunto de danzas america- } \\
\text { nas que se les ha enseñado. } \\
\text { - Cada grupo ha escrito los pasos } \\
\text { para elaborar su plato favorito ex- } \\
\text { poniéndolos a todos los grupos. } \\
\text { Estas exposiciones han generado } \\
\text { preguntas de unos a otros. } \\
\text { - Se ha mostrado gran imaginación } \\
\text { en la representación de un cuento } \\
\text { con sombras chinas. Se ha refleja- } \\
\text { do una gran implicación de todos } \\
\text { los niños. }\end{array}$ \\
\hline
\end{tabular}

- Para el objetivo específico 2.2:

- El niño ha tenido interés por conocer al personaje invitado preguntándole acerca de su mundo y ofreciéndole ayuda para que se quedara en la Tierra. 
- Ha tenido un trato amable.

- Para el objetivo específico 3.1:

- El niño ha situado correctamente los diferentes continentes a lo largo de todo el proceso de la actividad.

- El niño ha relacionado las actividades a su correspondiente continente.

- Para el objetivo específico 3.2:

- El niño se ha mostrado activo y participativo a la hora de bailar.

- El niño ha mostrado interés por aprender los pasos característicos de cada danza.

- Para el objetivo específico 3.3:

- El niño ha seguido un orden a la hora de elaborar una receta culinaria

- El niño ha dado originalidad a su receta

- Para el objetivo específico 3.4:

- La historia se ha relacionado con las marionetas confeccionadas

- La historia ha constado de coherencia siguiendo una introducción, nudo y desenlace.

En la tabla 2 se pueden observar los resultados que obtuvo cada sujeto en referencia a los objetivos específicos y sus criterios, la puntuación de los cuales tiene el siguiente significado:

- 0: El niño no ha logrado el objetivo.

- 1: El niño ha logrado con dificultad el objetivo.

- 2: El niño ha logrado el objetivo.

Los resultados (medias) de cada sujeto respeto a los objetivos generales los encontramos expuestos en la tabla 3 .

Como conclusión y a partir de los resultados obtenidos en las tablas 2 y 3 podemos decir que a nivel general todos los sujetos han conseguido los objetivos excepto dos sujetos recién llegados (sujeto 3, sujeto 6), que han tenido dificultades para superarlos, especialmente el objetivo 1 en el que se trata de fomentar la cohesión y cooperación de grupo mediante el dialogo y el respeto. Esto nos puede indicar que aunque los sujetos puedan sentirse interesados y abiertos por aprender, respetar y aceptar otras culturas (así lo vemos en los resultados, tabla 3 , que estos mismos sujetos obtienen en el objetivo $n^{\circ} 2$ y 3 relacionados con este último aspecto), su condición de inmigrante (recién llegado) les dificulta poder integrarse en el grupo por falta de desarrollo del lenguaje o más concretamente del idioma autóctono que están aprendiendo.

Por otro lado apreciamos la presencia de un sujeto (sujeto 1 en este caso autóctono) que asume dentro del grupo un rol de liderazgo negativo que le impide conseguir los objetivos (objetivo 1 especialmente) al presentar una actitud cerrada.

En cuanto a la valoración de los objetivos podemos decir que el número 2 es aquel que ha obtenido un mejor resultado y esto puede ser debido a que el origen de las familias (Marruecos) ayuda a estos niños tomar concienciación de la existencia de varias culturas que deben aceptarse y respetarse para poder convivir. 
TABLA 2: Puntuaciones de cada sujeto respeto a los objetivos específicos y sus criterios de evaluación.

\begin{tabular}{|c|c|c|c|c|c|c|c|c|c|}
\hline $\begin{array}{l}\text { Objetivos } \\
\text { generales }\end{array}$ & $\begin{array}{l}\text { Objetivos } \\
\text { específicos }\end{array}$ & Crietrios & $\begin{array}{c}\text { Sujeto } \\
1\end{array}$ & $\begin{array}{c}\text { Sujeto } \\
2\end{array}$ & $\begin{array}{c}\text { Sujeto } \\
3\end{array}$ & $\begin{array}{c}\text { Sujeto } \\
4\end{array}$ & $\begin{array}{c}\text { Sujeto } \\
5\end{array}$ & $\begin{array}{c}\text { Sujeto } \\
6\end{array}$ & $\begin{array}{c}\text { Sujeto } \\
7\end{array}$ \\
\hline \multirow{4}{*}{1} & \multirow{2}{*}{1.1} & $\mathrm{C} 1$ & 1 & 2 & 0 & 2 & 2 & 0 & 2 \\
\hline & & $\mathrm{C} 2$ & 0 & 1 & 0 & 2 & 2 & 0 & 1 \\
\hline & \multirow{2}{*}{1.2} & $\mathrm{C} 1$ & 2 & 2 & 1 & 2 & 2 & 1 & 2 \\
\hline & & $\mathrm{C} 2$ & 0 & 1 & 1 & 2 & 2 & 1 & 2 \\
\hline \multirow{4}{*}{2} & \multirow{2}{*}{2.1} & $\mathrm{C} 1$ & 1 & 1 & 1 & 2 & 2 & 1 & 2 \\
\hline & & $\mathrm{C} 2$ & 2 & 2 & 1 & 2 & 2 & 1 & 2 \\
\hline & \multirow{2}{*}{2.2} & $\mathrm{C} 1$ & 2 & 2 & 1 & 1 & 1 & 1 & 1 \\
\hline & & $\mathrm{C} 2$ & 2 & 2 & 2 & 2 & 2 & 2 & 2 \\
\hline \multirow{8}{*}{3} & \multirow{2}{*}{3.1} & $\mathrm{C} 1$ & 2 & 2 & 1 & 2 & 2 & 1 & 2 \\
\hline & & $\mathrm{C} 2$ & 2 & 2 & 2 & 2 & 2 & 2 & 2 \\
\hline & \multirow{2}{*}{3.2} & $\mathrm{C} 1$ & 2 & 2 & 2 & 1 & 1 & 2 & 1 \\
\hline & & $\mathrm{C} 2$ & 1 & 1 & 1 & 2 & 2 & 0 & 2 \\
\hline & \multirow{2}{*}{3.3} & $\mathrm{C} 1$ & 2 & 1 & 0 & 2 & 2 & 0 & 2 \\
\hline & & $\mathrm{C} 2$ & 1 & 1 & 1 & 2 & 2 & 1 & 1 \\
\hline & \multirow{2}{*}{3.4} & $\mathrm{C} 1$ & 2 & 2 & 2 & 2 & 2 & 1 & 2 \\
\hline & & $\mathrm{C} 2$ & 0 & 0 & 0 & 2 & 2 & 1 & 1 \\
\hline Resultados & & & 1,37 & 1,62 & 1 & 1,87 & 1,87 & 0,93 & 1,69 \\
\hline
\end{tabular}

TABLA 3: Medias de cada sujeto respeto a cada uno de los objetivos generales.

\begin{tabular}{|l|c|c|c|c|c|c|c|c|}
\hline & Sujeto 1 & Sujeto 2 & Sujeto 3 & Sujeto 4 & Sujeto 5 & Sujeto 6 & Sujeto 7 & $\begin{array}{c}\text { Mediana } \\
\text { total }\end{array}$ \\
\hline Objetivo 1 & 0.75 & 1.5 & 0.5 & 2 & 2 & 0.5 & 1.75 & 1.28 \\
\hline Objetivo 2 & 1.75 & 1.75 & 1.25 & 1.25 & 1.75 & 1.25 & 1.75 & 1.53 \\
\hline Objetivo 3 & 1.50 & 1.4 & 1.12 & 1.87 & 1.87 & 1 & 1.62 & 1.48 \\
\hline
\end{tabular}

En segundo lugar, el objetivo $\mathrm{n}^{\circ} 3$ es el mas cercano al objetivo numero 2 pero obtiene una menor puntuación debido a que requiere un mayor esfuerzo intelectual (hemos de pensar que la mayoría de sujetos a lo que se aplicó nuestro proyecto se encontraban en una situación de fracaso escolar).

El objetivo $n^{\circ} 1$ es el que ha obtenido menor puntuación debido a que los sujetos recién llegados provocan un descenso de la media. Esto nos hace pensar que deberían potenciarse en el conjunto de actividades realizadas a lo largo de nuestra experiencia más aspectos que facilitaran la cohesión de grupo para conseguir unos mejores resultados en los objetivos 2 y 3. 
A continuación presentaremos una valoración de carácter más cualitativo:

- Considerando nuestro proyecto como material curricular creemos que recoge la utilización de materiales diversos (manipulativos, orales, escritos, auditivos, etc.). Es el caso de SOS Racismo el cual realiza un conglomerado con estos recursos. Aspecto que también es fomentado por Coepfert (1995), Babí et al. (1997) e Intermón (1998. Motivo por el cual podemos considerarlo enriquecedor y flexible puesto que permite añadir o variar contenidos según el interés del educador.

- En cuanto a los objetivos y contenidos del proyecto podemos decir que existe cierto equilibrio entre los que son de carácter conceptual, actitudinal y procedimental a la vez que se relaciona con el entorno donde se desarrolla la actividad y se incluye la diversidad cultural como contenido, dotando todo ello de coherencia a nuestro proyecto.

Hemos de decir que la experiencia de nuestro proyecto también sufre algunas carencias:

- El número de sujetos de la muestra no es representativo.

- La temporalización de la actividad resultó ser escasa, por lo que consideramos que debería aumentar el tiempo de aplicación ya que éste va acompañado de una serie de factores externos que influyen en el resultado. Aún así cabe decir que el aumento del tiempo también puede perjudicar el rendimiento y aprendizaje de los sujetos.

- Deberíamos desarrollar una serie de estrategias que facilitaran un mayor acercamiento de aquellos usuarios que se mostraban más distantes al realizar la actividad (los inmigrantes recién llegados).

Aunque en el mundo de la interculturalidad ya existan múltiples experiencias que siguen una línea de trabajo similar a la nuestra creemos que nuestro proyecto resulta igualmente positivo debido a la actualidad del tema. Y aún así todavía falta mucho por andar. Por este motivo, creemos que desde las instituciones deberían promocionarse más proyectos de este carácter. Esta sensibilización en cuanto a las instituciones también la demuestran Roquer C. et al. (1997).

Por otro lado, tenemos que decir que aunque nuestra experiencia se ha llevado a cabo en el ámbito no formal, su flexible naturaleza permite que ésta pueda aplicarse también en un ámbito formal como eje transversal de las diferentes áreas que configuran el currículo escolar, como por ejemplo en el área de sociales, en la que se trabajan como contenido los flujos migratorios. En este sentido creemos que actualmente la multiculturalidad en las escuelas se trabaja de forma muy puntual, y dada la importancia que ésta va adquiriendo en nuestra sociedad, creemos que nuestra experiencia y material pueden ser útiles a la hora de aportar ideas para trabajarla.

Finalmente, nuestra actividad facilita la posibilidad de mejora a aquellas personas en situación de inadaptación con la ayuda de educadores y la sociedad en general, aunque dada la situación actual vemos que los esfuerzos que han de realizar los colectivos inmigrantes para salir de una situación precaria son más elevados que los que debe realizar cualquier niño/a.

\section{Referencias bibliográficas}

Aguilera et al. (1997): «De viaje con Mayra». Cuadernos de Pedagogía, no 264, pp. 81-86. Aula d'Esplai del Vallès. (2000). Contes. Monogràfic de tècniques d'animació. Sabadell: Author. 
Babí et al. (1997): «¿Y tu cómo lo ves?». Cuadernos de Pedagogía, no 264, pp. 74-77.

Balboas, P. (1995): Vols donar la volta al món? Fitxes de treball per als alumnes. Sta. Coloma de Gramenet: Casal del Mestre.

Clapés, A. et al. (1995): El Set-testes. Gimkana del civisme. Barcelona: Departament de Benestar Social.

Coepfert, J. (1995): Viaje a los países de los mil aromas. Los niños cocinan. París: Fédération artisans du monde.

Fundació Akwaba. (1999): Un Conte per a viatjar pel món. Barcelona: Ludoteca Apatam.

García, G. y Ramírez, J. (1996): Diseño y evaluación de proyectos sociales. Zaragoza: Libros Certeza Intermón (1988): Des de Bolivia: Música i Folklore [K7]. Barcelona: Author.

Intermón (1991): Pobles i llegendes; taller de teatre. Barcelona: Author.

Intermón. (1998): Àfrica al Sud del Sàhara (Primaria i Secundaria). Barcelona: Ed. Grafimeo.

Lasa, B. (1997): «Cuentos con gitanos». Cuadernos de Pedagogía, n 264, pp. 87-89

López, G. (2000): El Llibre dels contes del món. Barcelona: Ed. Integral.

Luque i Fidel (1998): Interculturalitat i educació una reflexió sociològica sobre l'acció educativa. Barcelona:

Marín, I. et al. (1999): “Un món de jocs... a l'Eixample”. Àfrica. Barcelona: Ed. Marinva, Serveis i projectes.

Mille, J. (1997): Manual bàsic per a l'elaboració de projectes. Barcelona: Agència Municipal de Serveis.

Molina, F. (1998): Interculturalitat i educació una reflexió sociològica sobre l'acció educativa. Lleida: Universitat de Lleida.

Movibaix. (1992): Ciutadans del Món. Sant Feliu de Llobregat: Author.

Palom, F. y Tort, L. (1991): Management en organizaciones al servicio del progreso humano. Madrid: Espasa-Calpe y CDN.

Ricart, M., Rial, R., Dolci, M., Rufí, J. i Ricart, R (1985): Els titelles a l'escola. Barcelona: Eumo.

Rodríguez, A., Gines, B. i Saliba, G. (1995): Qui Són Els àrabs? Catalunya: Ed. Departament d'Ensenyament.

Roquer, J et al. (1997): «Tres unidades para conocernos mejor». Cuadernos de Pedagogía, n 264, pp. 78-80.

Tort, L i Millán, M (2000): Disseny, desenvolupament i innovació del currículum. Barcelona: Universitat de Barcelona.

http://www.sosracisme.org

http://www.fuhem.es/CIP/EDUCA/intercul

http://www.xtec.es/recursos

http://www.pangea.org/edualter

Fecha de recepción: 04-03-02

Fecha de revisión: 12-06-03

Fecha de aceptación: 27-10-03 